\title{
Pacific
}

Journal of

Mathematics

\section{VELOCITY MAPS IN VON NEUMANN ALGEBRAS}

L. J. BUnCE AND John DaVid MaItLAND WRight 


\title{
VELOCITY MAPS IN VON NEUMANN ALGEBRAS
}

\author{
L.J. BUNCE AND J.D. MAITLAND WRIGHT
}

When $A$ is a $C^{*}$-algebra, a function $d: A_{s a} \rightarrow A_{s a}$ is said to be a velocity map if, for each commutative subalgebra $B$ of $A_{s a}, d: B \rightarrow A_{s a}$ is a derivation.

Let $A$ be a norm closed ideal, or quotient, in a von Neumann algebra without Type $I_{2}$ part and let $P(A)$ be the set of projections in $A$. It is shown that if $d: P(A) \rightarrow A$ is a bounded function such that $d(e f)=e d(f)+d(e) f$ whenever ef $=f e$, then $d$ extends uniquely to a derivation of $A$. Hence every velocity map of $A_{s a}$ bounded on the unit ball extends to a derivation of $A$.

Let $A_{s a}$ denote the self-adjoint part of a $C^{*}$-algebra $A$. A function $d$ : $A_{s a} \rightarrow A_{s a}$ is said to be a velocity map if

(i) $d(\lambda x)=\lambda d(x), \quad$ for all $\lambda \in \mathbb{R}, x \in A_{s a}$

(ii) $\quad d(x+y)=d(x)+d(y), \quad$ for all $x, y \in A_{s a}$ with $x y=y x$

(iii) $\quad d(x y)=x d(y)+d(x) y, \quad$ for all $x, y \in A_{s a}$ with $x y=y x$.

Velocity map are motivated by their relevance to quantum dynamics. Briefly, $A_{s a}$ is identified with the bounded observables (of a physical system) the rate of change of which is measured by the velocity maps on $A_{s a}$; only when two observables commute are they simultaneously measurable and is their product again an observable. See [4] for more details.

The point of interest and natural question is: When do velocity maps extend to derivations?

It transpires that linear velocity maps always extend to derivations (see Proposition 1). If linearity is not assumed then we are still able to obtain positive results for a large class of $C^{*}$-algebras that includes Calkin algebras and all von Neumann algebras without Type $I_{2}$ part. In fact we prove something more.

Given a $C^{*}$-algebra, $A$, we let $P(A)$ denote the set of projections of $A$. Our main result is the following.

Let $M$ be a von Neumann algebra without Type $I_{2}$ part and let $A$ be a norm closed ideal of $M$ or a quotient of a norm closed ideal of $M$. Let $d: P(A) \rightarrow A$ be any bounded function satisfying

$$
d(e f)=d(e) f+e d(f), \text { whenever } e f=f e .
$$


Then $d$ extends uniquely to a derivation of $A$.

We will show by example that the exclusion of Type $I_{2}$ algebras from the above statement is necessary.

By an ingenious direct constructive argument Rajarama Bhat [4, Theorem $3.5]$ proved that every linear velocity map on $B(H)_{s a}$ extends to a derivation of $B(H)$ when $H$ is separable. We need to show that this extension theorem holds for all $C^{*}$-algebras. In fact, we have:

Proposition 1. Let $B$ be any $C^{*}$-algebra and let $d: B_{s a} \rightarrow B$ be a (real) linear map such that

$$
d(x y)=d(x) y+x d(y), \text { whenever } x y=y x .
$$

Then $d$ extends to a derivation of $B$.

Proof. Recall that the Jordan product $a \circ b$ of $a, b \in B$ is given by $a \circ b=$ $\frac{1}{2}(a b+b a)$. So, $d\left(a^{2}\right)=2 d(a) \circ a$, for each $a \in B_{s a}$.

Given $x, y \in B_{s a}$ we have

$$
\begin{aligned}
d(x \circ y) & =\frac{1}{2}\left[d\left((x+y)^{2}\right)-d\left(x^{2}\right)-d\left(y^{2}\right)\right] \\
& =(d(x+y)) \circ(x+y)-d(x) \circ x-d(y) \circ y \\
& =d(x) \circ y+d(y) \circ x .
\end{aligned}
$$

By a simple calculation we see that

$$
\bar{d}(x \circ y)=\bar{d}(x) \circ y+x \circ \bar{d}(y), \text { for all } x, y \in B,
$$

where $\bar{d}: B \rightarrow B$ is the complex linear extension of $d$. In other words, $\bar{d}$ is a Jordan derivation of $B$ and hence, by a result of Sinclair [6], is a derivation of $B$ as required.

In all that follows $M$ is a von Neumann algebra without Type $I_{2}$ direct summand and $I$ is a norm closed two sided ideal of $M$. We let $I_{0}$ denote the norm closed two sided ideal of $I$ generated by the abelian projections in $I$. (A projection $e$ of $M$ is abelian, or Type $I_{1}$, if the algebra $e M e$ is abelian.)

We will need an extension of the solution of the Mackey-Gleason Problem given by the authors in $[\mathbf{1}],[\mathbf{2}]$ and summarised in $[\mathbf{3}]$. By way of preparation we note that if $\Omega$ is a maximal abelian $C^{*}$-subalgebra of $I$, then $\Omega=I \cap \bar{\Omega}$ and so is an ideal of $\bar{\Omega}$, the (abelian) weak closure of $\Omega$ in $M$. By spectral theory $\Omega$ is (as therefore is $I$ ) the norm closed linear span of its projections. Given $e, f \in P(M)$ with $e \leq f$ and $f \in P(I)$ we have $e \in P(I)$. For arbitrary $e, f \in P(I)$ we have $e \vee f-e \sim f-e \wedge f$. 
A projection $e$ of $M$ is said to be a Type $I_{n}$ projection, where $n<\infty$, if the von Neumann algebra $e M e$ is of Type $I_{n}$.

Lemma 1. Let $e_{1}, \ldots, e_{n}$ be Type $I_{3}$ projections in $M$ and let $p$ be a (possibly zero) abelian projection in $M$. Then $(e \vee p) M(e \vee p)$ (and hence eMe) has no Type $I_{2}$ direct summand, where $e=e_{1} \vee \ldots \vee e_{n} \vee p$.

Proof. If $(e \vee p) M(e \vee p)$ has a Type $I_{2}$ direct summand, then $z(e \vee p)=$ $\left(z e_{1}\right) \vee \ldots \vee\left(z e_{n}\right) \vee(z p)$ is a Type $I_{2}$ projection for some central projection $z$ of $M$. Since for $i=1, \ldots, n e_{i} M e_{i}$ has no Type $I_{2}$ direct summand this means that $z e_{i}=0$ so that $z(e \vee p)=z p$ is abelian, which is a contradiction.

\section{Lemma 2.}

(i) $P(I)$ is an increasing approximate unit of $I$.

(ii) If $M$ has no Type $I_{1}$ direct summand (as well as no Type $I_{2}$ direct summand), then

$\left\{e_{1} \vee \ldots \vee e_{n}: e_{i}\right.$ is a Type $I_{3}$ projection in $\left.I, i=1, \ldots n, n \in \mathbb{N}\right\}$

is an increasing approximate unit of $I_{0}$.

Proof.

(i) Note that $P(I)$ is an increasing net in $I$. Let $x$ be in $I$, where $x=x^{*}$, and let $\epsilon>0$. By the preamble there exist real numbers $\lambda_{1}, \ldots, \lambda_{n}$ and mutually orthogonal projections $e_{1}, \ldots, e_{n}$ in $I$ such that $\| x-$ $\sum_{1}^{n} \lambda_{i} e_{i} \|<\epsilon$. So, $\|x(1-p)\|<\epsilon$ where $p=\sum_{1}^{n} e_{i}$. If $q \in P(I)$ with $p \leq q$, then

$$
\|x(1-q)\|=\|x(1-q) x\|^{\frac{1}{2}} \leq\|x(1-p) x\|^{\frac{1}{2}}=\|x(1-p)\|<\epsilon .
$$

(ii) Suppose that $M$ has no Type $I_{1}$ direct summand and let $p$ be an abelian projection of $M$ contained in $I$ and so in $I_{0}$. Because $M$ has no Type $I_{2}$ direct summand either, there exist projections $q$ and $h$ in $M$ such that $p, q, h$ are mutually orthogonal and equivalent. The projection $e=p+q+h$ is then a Type $I_{3}$ projection in $I$ dominating $p$. We note that $e$ is in $I_{0}$.

For any projection $p$ in $I_{0}, p M p$ is a postliminal $C^{*}$-algebra because $I_{0}$ is postliminal as follows from $[\mathbf{5}, \S 6.1]$ for example. So $p M p$ is a direct sum of finitely many Type $I_{k}$ von Neumann algebras for certain $k<\infty$ and hence $p=p_{1}+\cdots+p_{r}$ for certain abelian projections $p_{i}$ in $I_{0}$. So $p \leq e_{1} \vee \ldots \vee e_{r}$ from some Type $I_{3}$ projections $e_{1}, \ldots, e_{r}$ of $I_{0}$ by the above.

Since, by (i), $P\left(I_{0}\right)$ is an increasing approximate unit for $I_{0}$ it now follows that the increasing net in $P\left(I_{0}\right)$ described in the statement is also an approximate unit of $I_{0}$. 
In the case when $A=M$, the following proposition is [2, Theorem A]. See also [3].

Proposition 2. Let the $C^{*}$-algebra $A$ be equal to a quotient of $I$, where $I$ is a norm closed ideal of $M$, and let $X$ be a Banach space. Let $\rho: P(A) \rightarrow X$ be a bounded function such that

$$
\rho(e+f)=\rho(e)+\rho(f), \quad \text { whenever } \text { ef }=0 .
$$

Then $\rho$ extends uniquely to a continuous linear map $\bar{\rho}: A \rightarrow X$.

Proof. (a) Let $A=I$. Suppose first that $X=\mathbb{C}$.

Because the maximal abelian subalgebras of $I$ are generated by projections we have, precisely as in $[1, \S 1]$, that $\rho$ extends uniquely to a function $\bar{\rho}: I \rightarrow$ $\mathbb{C}$ bounded on the unit ball of $I$ satisfying $\bar{\rho}(a+i b)=\bar{\rho}(a)+i \bar{\rho}(b)$ wherever $a=a^{*}$ and $b=b^{*}$, and which is linear on every abelian $C^{*}$-subalgebra of $I$. We make the harmless normalising assumption that $\bar{\rho}$ sends the unit ball of $I$ into the unit disc. We show that $\bar{\rho}$ is linear.

If $M$ has Type $I_{1}$ direct summand $z M$, where $z$ is central projection of $M$, then $I=z I \oplus(1-z) I$ and $\bar{\rho}$ is already linear on $z I$. Passing to $(1-z) I$, we may therefore suppose that $M$ has no Type $I_{1}$ direct summand (as well as having no Type $I_{2}$ direct summand).

We show first that $\rho$ is linear on $I_{0}$. Invoking Lemmas 1 and 2 we can an increasing approximate unit $\left(e_{\lambda}\right)$ of $I_{0}$ contained in $\rho\left(I_{0}\right)$ for which the algebras $e_{\lambda} M e_{\lambda}$ and $\left(e_{\lambda} \vee p\right) M\left(e_{\lambda} \vee p\right)$ have no Type $I_{2}$ direct summand, for all $\lambda$ and for all abelian projections $p$ in $I_{0}$. Consequently, $\bar{\rho}$ is linear on all of these algebras by $[2$, Theorem A].

Let $x$ be a self-adjoint element of $I_{0}$ and let $\epsilon>0$. There is a $\lambda_{0}$ such that $\left\|e_{\lambda} x e_{\lambda}-e_{\mu} x e_{\mu}\right\|<\epsilon$, for all $\lambda, \mu \geq \lambda_{0}$. Linearity of $\bar{\rho}$ on $\left(e_{\lambda} \vee e_{\mu}\right) M\left(e_{\lambda} \vee e_{\mu}\right)$ implies that $\left|\bar{\rho}\left(e_{\lambda} x e_{\lambda}\right)-\bar{\rho}\left(e_{\mu} x e_{\mu}\right)\right|<\epsilon$, for all $\lambda, \mu>\lambda_{0}$. Consequently, $\rho\left(e_{\lambda} x e_{\lambda}\right)$ converges for all $x$ in $I_{0}$ and we see that the map $\tau: A \rightarrow \mathbb{C}$ defined by $\tau(x)=\lim \bar{\rho}\left(e_{\lambda} x e_{\lambda}\right)$ is linear.

When $p$ is an abelian projection in $I_{0}$, linearity of $\bar{\rho}$ on all $\left(e_{\lambda} \vee p\right) M\left(e_{\lambda} \vee p\right)$ and the fact that $\left\|e_{\lambda} p e_{\lambda}-p\right\|<\epsilon$ for all large enough $\lambda$, gives $\rho(p)=$ $\lim \bar{\rho}\left(e_{\lambda} p e_{\lambda}\right)$ by similar reasoning. Hence $\rho(e)=\tau(e)$ for all $e \in P\left(I_{0}\right)$, because every projection of $I_{0}$ is a finite orthogonal sum of abelian projections in $I_{0}$. It follows that $\bar{\rho}$ agrees with $\tau$ on all of $I_{0}$ and so is linear there.

Now let $e$ be any projection in I. We have $e M e=M_{1} \oplus M_{2}$, where $M_{1}$ is Type $I_{2}$ or zero and $M_{2}$ has no Type $I_{2}$ direct summand. But then $M_{1} \subset I_{0}$ and so $\bar{\rho}$ is linear on $M_{1}$ by the above. By [2, Theorem A], $\rho$ is linear on $M_{2}$. Hence $\bar{\rho}$ is linear on $e M e$, for all $e \in P(I)$. Now, repeating appropriately the arguments of the previous paragraf, this time for the approximate unit $P(I)$ of $\mathrm{I}$, we have that $\bar{\rho}$ is linear on $\mathrm{I}$. 
The general case, for an arbitrary Banach space $\mathrm{X}$, now follows as in Lemma 1.1. of [2] or [3].

(b) Let $A=I / J$, where $\mathrm{J}$ is a norm closed two-sided ideal of $\mathrm{I}$ and let $\pi: I \rightarrow I / J$ be the canonical homomorphism. By the above, $\rho \pi: P(I) \rightarrow X$ then extends to a continuous linear map $\varphi: I \rightarrow X$ vanishing on $\mathrm{J}$. The induced linear map $\bar{\varphi}: I / J \rightarrow X$ extends $\rho$ (uniquely) as desired. This completes the proof.

We can now prove our main result.

Theorem . Let $M$ be a von Neumann algebra without Type $I_{2}$ part and let $A$ be a norm closed ideal of $M$ or be a quotient of a norm closed ideal of $M$. Let $d: P(A) \rightarrow A$ be a bounded function such that

$$
d(e f)=d(e) f+e(d f), \quad \text { whenever } \text { ef }=f e .
$$

Then $d$ extends uniquely to a derivation of $A$.

Proof. We note that $d(p)=d(p) p+p d(p)$, for all projections $p$ of $A$. Let $e$ and $f$ be orthogonal projections of $A$. Thus, as $e f=f e=0$, we have

$$
d(e) f+e d(f)=d(f) e+f d(e)=0 .
$$

In addition, since $e=e(e+f)=(e+f) e$, we have

$$
d(e)=d(e)(e+f)+e d(e+f)=(d(e+f)) e+(e+f) d(e),
$$

so that

$$
2 d(e)=e d(e+f)+(d(e+f)) e+d(e)(e+f)+(e+f) d(e),
$$

and hence, using $\left(^{*}\right)$

$$
d(e)=e d(e+f)+(d(e+f)) e+d(e) f+f d(e) .
$$

Similarly,

$$
d(f)=f d(e+f)+(d(e+f)) f+d(f) e+e d(f) .
$$

The final two equations, together with $\left({ }^{*}\right)$, imply that

$$
d(e)+d(f)=(e+f) d(e+f)+(d(e+f))(e+f)=d(e+f) .
$$

Therefore, by Proposition 2, $d$ extends uniquely to a continuous linear map $\bar{d}: A \rightarrow A$. It follows that the assignment

$$
h(x, y)=\bar{d}(x y)-\bar{d}(x) y-x \bar{d}(y)
$$


is continuous and bilinear on $A \times A$. Let $\Omega$ be an abelian $C^{*}$-subalgebra of A. By assumption $h(e, f)=0$ for all projections $e, f$ in $\Omega$. But $\Omega$ is the norm closed linear span of its projections. Hence, by continuous bilinearity, $h$ is identically zero on $\Omega \times \Omega$. Therefore,

$$
\bar{d}(x y)=\bar{d}(x) y+x \bar{d}(y), \text { for all } x, y \text { in } A_{s a} \text { with } x y=y x .
$$

Hence, by Proposition $1, \bar{d}$ is a derivation.

Corollary . Let $A$ be as in the Theorem and let $d: A_{s a} \rightarrow A_{s a}$ be a velocity map bounded on the ball of $A_{s a}$. Then d extends to a derivation of $A$.

The Theorem and its Corollary apply to all von Neumann algebras without Type $I_{2}$ direct summand and to their norm closed two sided ideals and quotients. So, for instance, they apply to the Calkin algebra and also to every dual $C^{*}$-algebra without Type $I_{2}$ representations. But the exclusion of Type $I_{2}$-algebras is necessary. The following example explains why.

Example. Let $M=M_{2}(\mathbb{C})$.

Let $p=\left[\begin{array}{ll}1 & 0 \\ 0 & 0\end{array}\right], u_{n}=\left[\begin{array}{cc}\cos \left(\theta_{n}\right) & \sin \left(\theta_{n}\right) \\ -\sin \left(\theta_{n}\right) & \cos \left(\theta_{n}\right)\end{array}\right]$ and $p_{n}=u_{n} p u_{n}^{*}$ where $\theta=\pi / 5^{n}$, for all $n \in \mathbb{N}$. Notice that the $p_{n}$ are mutually non-orthogonal and that

$$
\bar{p}_{n}=p_{n} \bar{p}_{n}+\bar{p}_{n} p_{n}, \quad \text { where } \bar{p}_{n}=u_{n}\left[\begin{array}{cc}
0 & (-1)^{n} \\
(-1)^{n} & 0
\end{array}\right] u_{n}^{*},
$$

for all $n \in \mathbb{N}$. Define a bounded function, $d: P(M) \rightarrow M_{s a}$, by

$$
\begin{gathered}
d\left(p_{n}\right)=\bar{p}_{n}, \text { for all } n \in \mathbb{N} \\
d\left(1-p_{n}\right)=-\bar{p}_{n}, \text { for all } n \in \mathbb{N}
\end{gathered}
$$

$d(q)=0$, whenever $q$ is not in $\left\{p_{n}: n \in \mathbb{N}\right\} \cup\left\{1-p_{n}: n \in \mathbb{N}\right\}$.

Since commuting non-zero projections are either equal or are orthogonal with sum 1 it is easy to see that $d$ satisfies

$$
\begin{gathered}
d(e f)=e d(f)+d(e) f, \text { whenever } \text { ef }=f e \\
d(e+f)=d(e)+d(f), \text { whenever } \text { ef }=0 .
\end{gathered}
$$

Also, by direct calculation, (or a simple version of arguments deployed in the proof of the Theorem) we observe that $d$ has a unique extension to a velocity $\operatorname{map} \bar{d}: M_{s a} \rightarrow M_{s a}$ bounded on the unit ball. But $\bar{d}$ is not linear because $d$ is discontinuous. For example, $p_{n} \rightarrow e$ whereas $d\left(p_{n}\right)$ fails to converge. So $d$ (and $\bar{d}$ ) cannot extend to a derivation of $M$. 


\section{References}

[1] L.J. Bunce and J.D. Maitland Wright, Complex measures on projections in von Neumann algebras, J. Lond. Math. Soc. To appear.

[2] The Mackey Gleason Problem for vector measures on projections in von Neumann algebras, J. Lond. Math. Soc. To appear.

[3] The Mackey-Gleason Problem, Bull. A.M.S., 26 (1992), 288-293.

[4] B.V. Rajarama Bhat, On a characterization of velocity maps in the space of observables, Pac. J. Math., 152 (1992), 1-14.

[5] G.K. Pedersen, $C^{*}$-algebras and their automorphism groups, Academic Press, 1979.

[6] A.M. Sinclair, Jordan homomorphisms and derivations on semi-simple Banach algebras, Proc. Amer. Math. Soc., 24 (1970), 209-214.

Received December 14, 1992 and revised June 11, 1993.

READING UNIVERSITY

READING RG6 2AX,

U.K.

E-MAIL ADDRESS: MATHS@READING.AC.UK

AND

IsaAC Newton Inst. For Math. Sciences

20 Clarkson RoAD

CAmbridge CB3 OEH

U.K.

E-MAIL ADDRESS: WRIGHT@NEWTON.CAM.AC.UK 



\section{CONTENTS}

N. Ben Amar, Tangential deformations on the dual of nilpotent special Lie

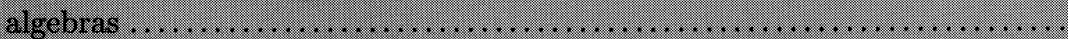

Martin Bendersky, Donald M. Davis and Mark Mahowald, $v_{1}$-periodic

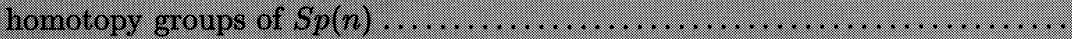

Georgia Benkart, Seok-Jin Kang, Kailash C. Misra, Indefinite Kac-

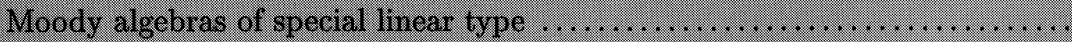

Robin Brooks and Charles Odenthal, Nielsen numbers for roots of maps

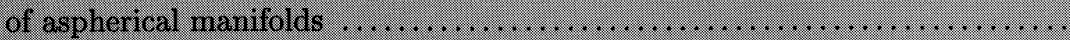

L.J. Bunce and J.D. Maitland Wright, Velocity maps in von Neumann

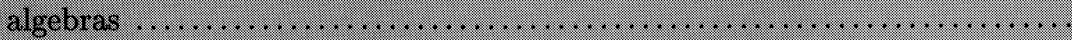

Bradley N. Currey, Smooth decomposition of finite multiplicity monomial representations for a class of completely solvable homogeneous spaces ...... R.J. Daverman and D.F. Snyder, On proper surjections with locally triv-

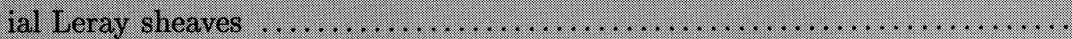

Patrick N. Dowling, Zhibao Hu and Mark A. Smith, MLUR renormings

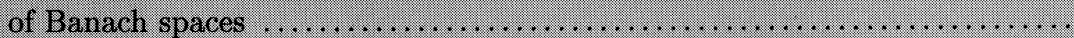
Tuval Foguel, Finite groups with a special 2-generator property ..........

Mourad E.H. Ismail and Mizan Rahman, Some basic bilateral sums and

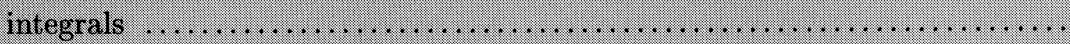

Wojciech Jaworski, Strong approximate transitivity, polynomial growth, and spread out random walks on locally compact groups $\ldots \ldots \ldots \ldots \ldots . . . . . .$.

N. Kutev and F. Tomi, Nonexistence and instability in the exterior Dirichlet problem for the minimal surface equation in the plane............

A. Nobile, Equisingularity Theory for Plane Curves With Embedded

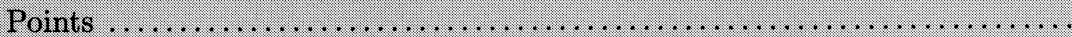

Dominikus Noll, Directional differentiability of the metric projection in Hilbert space 


\section{PACIFIC JOURNAL OF MATHEMATICS}

Volume $170 \quad$ No. $2 \quad$ October 1995

Tangential deformations on the dual of nilpotent special Lie algebras

297

NABIHA BEN AMAR

$v_{1}$-periodic homotopy groups of $S p(n)$

319

MaRTIN BENDERSKY, DONALD M. DAVIS and MARK MAHOWALD

Indefinite Kac-Moody algebras of special linear type

GeOrgia BenKart, SEOK-Jin KANG and KaILASh C. MisRa

Nielsen numbers for roots of maps of aspherical manifolds

405

ROBIN B. S. BROOKS and CHARLES ODENTHAL

Velocity maps in von Neumann algebras

L. J. BUNCE and JOHN DAVID MAITLAND WRIGHT

Smooth decomposition of finite multiplicity monomial representations for a class of 429 completely solvable homogeneous spaces

\section{BRADLEY CURREY}

On proper surjections with locally trivial Leray sheaves

ROBERT JAY DAVERMAN and DAVID FRED SNYDER

MLUR renormings of Banach spaces

PATRICK DOWLING, Zhibao Hu and MARK ANDREW SMIth

Finite groups with a special 2-generator property

TUVAL S. Foguel

Some basic bilateral sums and integrals

MOURAD ISMAIL and MIZAN RAHMAN

Strong approximate transitivity, polynomial growth, and spread out random walks on 517 locally compact groups

WOJCIECH JAWORSKI

Nonexistence and instability in the exterior Dirichlet problem for the minimal surface 535 equation in the plane

NiKolai KuteV and FriedRich TOMI

Equisingularity theory for plane curves with embedded points

Augusto Nobile

Directional differentiability of the metric projection in Hilbert space

DOMINIKUS NOLL 